\title{
An Apology from the Editors of OR Insight
}

Steve Clarke and Brian Lehaney

The September issue of OR Insight fell below the high standards that we have tried to set since we took over editorship of this publication. There were a number of unprecedented problems concerning general production of the journal, which gave rise to unacceptable typographical and formatting errors. We, the editors, would like to apologise to both authors and readers for these. We would like to make it clear that these shortcomings within the September issue are in no way attributable to the authors, and were faults incurred during the production process.
The published version of the Editorial, originally prepared by Professor Miles G. Nicholls of Swinburne University, Victoria, Australia, suffered significantly as a result. We are therefore pleased to reprint September's editorial in full on the following pages.

This incident has given us cause to be more vigilant in the future, and we will endeavour to ensure high quality for the future. We sincerely hope that you, our readers, will continue to enjoy OR Insight. 